\title{
PWE-079 DRINKING BEHAVIOUR AND RS738409:G IN PNPLA3 ARE ASSOCIATED WITH SLOWER RECOVERY OF LIVER FUNCTION FOLLOWING SEVERE ALCOHOLIC HEPATITIS
}

1 S Atkinson*, 2,3M Way, 3 A McQuillin, MY Morgan, 1 M Thursz. 1 Hepatology, Imperial College London; 2 Liver and Digestive Health, Department of Medicine; 3 Molecular Psychiatry, Faculty of Brain Sciences, University College London, London, UK 10.1136/gutjnl-2017-314472.325

Introduction The variant rs738409 in PNPLA3 is associated with an increased risk of developing both significant alcohol-related liver disease and, in this context, adverse clinical outcomes. The aim of this study was to determine whether, in severe alcoholic hepatitis, carriage of rs738409:G is associated with impaired recovery of liver function.

Method Cases with severe alcoholic hepatitis were recruited prospectively through the Steroids or Pentoxifylline for Alcoholic Hepatitis (STOPAH) trial. Biochemical and haematological data were collected at baseline and the day 90 visit. Self-reported alcohol consumption was recorded at day 90 and categorised as abstinent or drinking. The rates of change in serum bilirubin and albumin concentrations and the international normalised ratio (INR) were examined in relation to rs738490 genotype and drinking behaviour.

Results Paired information on rs738409 genotype and laboratory variables was available in 377 cases; information on drinking behaviour was available in 365 (97\%). Carriage of rs738409: $\mathrm{G}$ was associated with a slower decline in median serum bilirubin concentration (CC: $0.0084 \mathrm{mmol} / \mathrm{l} /$ day [IQR 0.0066-0.010]; CG: 0.0080 [0.0053-0.0098]; GG 0.0072 [0.0045-0.0092], $\mathrm{p}<0.001$ ) and a slower recovery of serum albumin concentrations and (CC: 0.0027 g/l/day [0.0009-0.006]; CG: 0.0025 [0.0005- 0.0032]; GG 0.0017 [0.00040.0035 ], $p=0.04$ ), but did not appear to influence recovery of the INR ( $\mathrm{p}=0.90$ (Figure 1). Resumption of drinking by day 90 significantly influenced the recovery rates of all three laboratory variables viz. serum bilirubin: (abstinent 0.0088 [0.0069-0.01] vs drinking 0.0067 [0.0026-0.009], serum albumin $(0.0027$ [0.0057-0.001] vs 0.0018 [0.00420.0003 ], $\mathrm{p}=0.007)$; and INR (0.0022 [0.0012-0.0034] vs 0.0013 [ $0.0005-0.0027], \mathrm{p}$ $<0.001$ ). The observed effect of rs 738409 genotype on the rates of recovery of laboratory variables differed in groups defined by drinking behaviour; recovery rates were slower in abstinent individuals who carried rs738409:G but rates were not further influenced by genotype in those who continued to drink

Conclusion In people who survive an acute episode of severe alcoholic hepatitis carriage of rs738409:G is associated with slower rates of recovery in serum bilirubin and albumin. Early resumption of drinking also significantly slows recovery of these and laboratory variables. The greatest influence of rs 738409: $\mathrm{G}$ was in relation to recovery rates in abstinent people.

Disclosure of Interest None Declared 